\title{
Search for cool white dwarfs with GSC2
}

\author{
D. Carollo, A. Spagna, M.G. Lattanzi, R.L. Smart
}

INAF-Osservatorio Astronomico di Torino, Strada Osservatorio 20

Pino Torinese I-10025, Italy

S.T. Hodgkin

Cambridge Astronomical Survey Unit, Institute of Astronomy, Madingley Road,Cambridge,CB3 OHA,UK

L. Terranegra

INAF-Osservatorio Astronomico di Capodimonte, Via Moiariello 16, I-80131 Napoli, Italy

B. McLean

Space Telescope Science Institute, 3700 San Martin Drive, Baltimore, MD 21218, USA

\begin{abstract}
Microlensing experiments have suggested that a significant part of the dark halo of the Milky Way could be composed of matter in the form of massive compact halo objects (MACHOs). Cool ancient white dwarfs (WDs) are the natural candidates. Here we present a new survey of halo WDs and evaluate the local space density using an accurate kinematic analysis. A comparison to a revaluation of the Oppenheimer et al. result is also provided. The local space density estimated for the two independent samples is about $\sim 10^{-5} \mathrm{M}_{\odot} \mathrm{pc}^{-3}$.
\end{abstract}

\section{Baryonic Dark Matter in the Galactic Halo}

Several candidates for the Baryonic Dark Matter such as brown dwarfs, planets, ancient cool white dwarfs, neutron stars and primordial black holes have been proposed. All these halo objects could be detected by gravitational microlensing (Pacynski 1986). The MACHO project results seem to indicate that $20 \%$ of the dark halo is made of compact objects with mass of $0.5 M_{\odot}$ (Alcock et al. 2000). The favoured candidates are very old cool WDs with $T_{\text {eff }}<4000 \mathrm{~K}$. The most extensive survey (Oppenheimer et al. 2001) claimed the discovery of 38 halo WDs and derived a local density of $1.6 \times 10^{-4} \mathrm{M}_{\odot} \mathrm{pc}^{-3}$ which corresponds to a fraction of $2 \%$ of the galactic dark halo density. Nevertheless, these results are still a matter of debate (see e.g., Torres et al. 2002 for a review). 


\section{GSC2 Survey: plate material and selection criteria}

Our survey covers an area of $\sim 1100$ sq-deg, mostly distributed toward the NGP. The material consists of digitized Schmidt plates from the Northern photographic surveys which have been utilized for the construction of the Guide Star Catalogue-II (McLean et al. 2000). We selected high proper motion $(\mu>0.3 \mathrm{as} / \mathrm{yr})$ and faint targets $\left(R_{F}<19.5\right)$ (Carollo et al. 2003a). The sample was refined by visual inspection and cross correlation with other catalogues. Finally, low resolution spectroscopy was performed to determine the spectral type and the main chemical composition of the stars. We used the 3.5 $\mathrm{m}$ TNG+DOLORES (La Palma) and the $4.2 \mathrm{~m}$ WHT+ISIS (La Palma).

\section{Analysis of the sample and preliminary results}

We obtained spectra for 50 stars and the total number of confirmed WDs is 35 , of which 14 are without an $\mathrm{H} \alpha$ line, including a few exotic objects like the coolest ever carbon rich WD (Carollo et al. 2003b). In order to derive the halo or thick disk membership of the confirmed WDs, we first evaluated the photometric parallaxes using a relation $M_{F}$ vs. $B_{J}-R_{F}$. The calibration was based on a sample of WDs with known trigonometric parallax (Bergeron et al. 2001) and adopting the colour transformations BVR $\Rightarrow B_{J} R_{F} I_{N}$ used for the GSC-II. We computed distances with an uncertainty of $25-30 \%$ including the cosmic dispersion and photometric errors. Also, our magnitude limited sample was corrected for the Malmquist bias. Then an accurate determination of the membership was performed in the tangential velocity plane $\left(V_{\alpha}, V_{\delta}\right)$ of each target, where we have projected the velocity distribution of a kinematically selected sample. The criterion for membership of each target is to select as a halo star those objects which are not consistent with the thick disk kinematics at the $99 \%$ confidence level. Finally, in order to evaluate the local space density (via the $1 / V_{\max }$ method) our analysis was applied both to the GSC-II and Oppenheimer et al. samples. The results are remarkable; in fact we found 2 halo WDs for the first sample (still preliminary) and only 7 halo WDs for the second sample (4165 sq-deg). The local space density in both cases is $\sim 10^{-5} \mathrm{M}_{\odot} \mathrm{pc}^{-3}$. This value is consistent with the local mass density of halo stars from Gould et al. (1998).

\section{References}

Alcock, C. et al. 2002, ApJ, 542, 281

Bergeron, P. et al. 1997, ApJS, 108, 339

Carollo, D. et al. 2003a, in ASP Conf. Ser. 298, 375

Carollo, D. et al. 2003b, A\&A, 400, L13

Gould, A. et al. 1998, ApJ, 499, 728

McLean, B. et al 2000, ASP Conf. Ser. 216, 145

Oppenheimer, B.R. et al. 2001, Science, 292, 698

Pacynski, B. 1985, ApJ, 304, 1

Torres, S. et al. 2002, MNRAS, 336, 971 